\title{
A Second Order Adjoint Method to Targeted Observations
}

\author{
Humberto C. Godinez and Dacian N. Daescu \\ Department of Mathematics and Statistics, \\ Portland State University, Portland OR 97207 \\ $\{$ hgodinez, daescu\}@pdx.edu
}

\begin{abstract}
The role of the second order adjoint in targeting strategies is studied and analyzed. Most targeting strategies use the first order adjoint to identify regions where additional information is of potential benefit to a data assimilation system. The first order adjoint posses a restriction on the targeting time for which the linear approximation accurately tracks the evolution of perturbation. Using second order adjoint information it is possible to maintain some accuracy for longer time intervals, which can lead to an increase on the target time. We propose the use of the dominant eigenvectors of the Hessian matrix as an indicator of the directions of maximal error growth for a given targeting functional. These vectors are a natural choice to be included in the targeting strategies given their mathematical properties.
\end{abstract}

\section{Introduction}

Observation targeting strategies aim to identify optimal regions where supplemental data can improve the forecast of a data assimilation system. Adjoint modeling has been an essential tool for the development of targeting strategies in the context of variational data assimilation methods. The adjoint of the tangent linear model associated to an atmospheric model is a key ingredient to implementing various targeting strategies, such as gradient sensitivity, dominant singular vectors, and sensitivity to observations ([1, [2, 3], 4], 5], 6]).

The first order adjoint (FOA) model provides the gradient of a scalar-valued forecast aspect, typically a forecast error measure. As such, the FOA represents a first order approximation to the evolution of perturbations in the atmospheric model. The accuracy of this approximation is limited by the magnitude of the perturbation and by the time length of the forecast. As the forecast time lead increases, the accuracy of the FOA to track the initial-condition error propagation is impaired. This poses a limitation on the time window for which targeting strategies based on the FOA fields are reliable. To overcome this practical difficulty and to increase the effectiveness of adjoint targeting strategies, a second order adjoint (SOA) model may be considered to capture the quadratic terms in the error growth approximation. An overview of the SOA model implementation and applications to variational data assimilation is provided in [7]. 
In our work a targeting strategy based on SOA modeling is considered and numerical experiments are presented in a comparative analysis between the first order and the second order adjoint-based observation targeting guidance. The importance of incorporating SOA information is investigated by using first and second order Taylor approximations to model the nonlinear error growth and perturbations in a forecast error functional.

Section 2 briefly revisits the four dimensional variational (4D-Var) data assimilation and the FOA and SOA models. In section 3 the implementation of the FOA and SOA models to a shallow water (SW) model is presented. First and second order Taylor approximations to the perturbations in a forecast error functional are analyzed. A novel targeting strategy based on the eigenvalues and eigenvectors of the Hessian matrix of the forecast aspect is implemented in section 4 using the SW model. Conclusions and future work are in section 5 .

\section{Data Assimilation and Adjoint Modeling}

Given an initial state $\mathbf{x}_{0}$, let $\mathcal{M}_{i}$ denote the discrete atmospheric model (forward model) that evolves the state from $t_{i}$ to $t_{i+1}$

$$
\mathbf{x}_{i+1}=\mathcal{M}_{i}\left(\mathbf{x}_{i}\right), \quad i=0, \ldots, N-1 .
$$

Data assimilation techniques [8] combine information from a dynamical model, a prior (background) estimate, and observational data to provide an optimal initial condition (analysis) to the dynamical system (10). The 4D-Var analysis [9] is obtained by minimizing a cost functional that measures the discrepancy between the model state, background estimate, and time distributed observational data

$$
\begin{aligned}
\mathcal{J}\left(\mathbf{x}_{0}\right)=\left(\mathbf{x}_{0}-\mathbf{x}^{b}\right)^{T} \mathbf{B}^{-1} & \left(\mathbf{x}_{0}-\mathbf{x}^{b}\right)+ \\
& \sum_{i=0}^{k}\left(\mathbf{y}_{i}-H_{i}\left[\mathbf{x}_{i}\right]\right)^{T} \mathbf{R}_{i}^{-1}\left(\mathbf{y}_{i}-H_{i}\left[\mathbf{x}_{i}\right]\right)
\end{aligned}
$$

where $\mathbf{x}^{b}$ is the background, $\mathbf{y}_{i}$ is the observation vector at $t_{i}, \mathbf{B}$ and $\mathbf{R}$ are the error covariance matrices for the background and observations, respectively, and $H_{i}$ is the observational operator mapping the state into observations at $t_{i}$.

Adaptive observations are supplementary data collected to reduce the error of some aspect of the forecast at verification time $t_{v}>t_{k}$ over a verification domain $\mathcal{D}_{v}$, expressed as

$$
\mathcal{J}_{v}\left(\mathbf{x}_{v}\right)=\frac{1}{2}\left\langle\mathbf{P}\left(\mathbf{x}_{v}-\mathbf{x}_{v}^{t}\right), \mathbf{P}\left(\mathbf{x}_{v}-\mathbf{x}_{v}^{t}\right)\right\rangle_{\mathbf{E}}
$$

where $\mathbf{x}_{v}^{t}$ is the true state at the verification time, $\mathbf{x}_{v}$ is the state of the system at time $t_{v}, \mathbf{P}$ is a projection operator on $\mathcal{D}_{v}$ satisfying $\mathbf{P}^{*} \mathbf{P}=\mathbf{P}^{2}=\mathbf{P}$. The inner product $\langle,\rangle_{\mathbf{E}}$ is defined as $\langle\mathbf{x}, \mathbf{y}\rangle_{\mathbf{E}}=\langle\mathbf{x}, \mathbf{E y}\rangle$, where $\mathbf{E}$ is a symmetric positive definite matrix, typically chosen to induce the total energy norm. The measure (3) is the forecast error functional at the verification time $t_{v}$ over the verification domain $\mathcal{D}_{v}$. 


\subsection{Taylor Expansion of the Forecast Error Functional}

The functional $\mathcal{J}_{v}$ implicitly depends on the initial condition $\mathbf{x}_{0}$ of (1)

$$
\mathcal{J}_{v}\left(\mathbf{x}_{N}\right)=\mathcal{J}_{v}\left(\mathcal{M}_{t_{0} \rightarrow t_{N}}\left(\mathbf{x}_{0}\right)\right)
$$

where $\mathcal{M}_{t_{0} \rightarrow t_{N}}$ is the nonlinear model integration from $t_{0}$ to $t_{N}=t_{v}$,

$$
\mathcal{M}_{t_{0} \rightarrow t_{N}}=\mathcal{M}_{N-1} \circ \cdots \circ \mathcal{M}_{0}\left(\mathbf{x}_{0}\right) \text {. }
$$

A perturbation $\delta \mathbf{x}_{0}$ in the initial condition will result in a perturbation $\delta \mathcal{J}_{v}\left(\mathbf{x}_{N}\right)=$ $\mathcal{J}_{v}\left(\mathbf{x}_{N}+\delta \mathbf{x}_{N}\right)-\mathcal{J}_{v}\left(\mathbf{x}_{N}\right)$ that, to a second order Taylor approximation, can be expressed

$$
\delta \mathcal{J}_{v}\left(\mathbf{x}_{N}\right) \approx \nabla_{\mathbf{x}_{0}} \mathcal{J}_{v}\left(\mathbf{x}_{N}\right) \delta \mathbf{x}_{0}+\frac{1}{2} \delta \mathbf{x}_{0}^{T} \nabla_{\mathbf{x}_{0}}^{2} \mathcal{J}_{v}\left(\mathbf{x}_{N}\right) \delta \mathbf{x}_{0}
$$

The gradient $\nabla_{\mathbf{x}_{0}} \mathcal{J}_{v}\left(\mathbf{x}_{N}\right)$ is obtained through the FOA model associated to (1)

$$
\begin{aligned}
\lambda_{N} & =\nabla_{\mathbf{x}_{N}} \mathcal{J}_{v}\left(\mathbf{x}_{N}\right) \\
\lambda_{i} & =\mathbf{M}_{i}^{*}\left(\mathbf{x}_{i}\right) \lambda_{i+1}, \quad i=N-1, \ldots, 0
\end{aligned}
$$

where $\mathbf{M}_{i}$ is the derivative (tangent linear model) of $\mathcal{M}_{i}$, and $\mathbf{M}_{i}^{*}$ its adjoint.

Second order derivative information, as the product of the Hessian $\nabla_{\mathbf{x}_{0}}^{2} \mathcal{J}_{v}\left(\mathbf{x}_{N}\right)$ times a user-defined vector, may be obtained by integration of a SOA model.

\subsection{SOA Model Equations}

The equations of the discrete SOA model associated to (1) and (4) are

$$
\begin{aligned}
\nu_{N} & =\nabla_{\mathbf{x}_{N}}^{2} \mathcal{J}_{v}\left(\mathbf{x}_{N}\right) \mu_{N}=\mathbf{P}^{T} \mathbf{E} \mathbf{P} \mu_{N} \\
\nu_{i} & =\mathbf{M}_{i}^{*}\left(\mathbf{x}_{i}\right) \nu_{i+1}+\frac{\partial}{\partial \mathbf{x}_{i}}\left[\mathbf{M}_{i}^{*}\left(\mathbf{x}_{i}\right) \bar{\lambda}_{i+1}\right] \mu_{i}, \quad i=N-1, \ldots, 0
\end{aligned}
$$

where $\mu$ is the solution to the tangent linear model (TLM)

$$
\begin{aligned}
\mu_{0} & =\mathbf{w} \\
\mu_{i+1} & =\mathbf{M}_{i}\left(\mathbf{x}_{i}\right) \mu_{i}, \quad i=0, \ldots, N-1,
\end{aligned}
$$

$\mathbf{w}$ is an user-defined vector, and the notation $\bar{\lambda}_{i+1}$ in the last term of (10) indicates that the state derivative applies to the $\mathbf{M}_{i}^{*}\left(\mathbf{x}_{i}\right)$ operator only while treating the adjoint variables $\lambda_{i+1}$ as constants $([10,7])$.

The solution of the SOA model (9)-(10) provides the Hessian vector product $\nabla_{\mathbf{x}_{0}}^{2} \mathcal{J}\left(\mathbf{x}_{0}\right) \mu_{0}=\nu\left(t_{0}\right)$ that is required to evaluate the second order term in the Taylor approximation (6), thus providing the quadratic term for the evolution of perturbations in the forward model. 


\section{The SW Model, FOA and SOA Taylor Approximations}

A global 2D shallow water (SW) model on a sphere is used for the numerical experiments. The model describes the hydrodynamic flow on a sphere under the assumptions that the vertical motion is much smaller that the horizontal motion. It is also assumed that the fluid depth is small compared with the radius of the sphere (radius of Earth). The equations of the SW model are

$$
\begin{aligned}
& \frac{\mathrm{d} \mathbf{v}}{\mathrm{d} t}=-f \mathbf{k} \times \mathbf{v}-\nabla \phi, \\
& \frac{\partial \phi}{\partial t}=-\nabla \cdot\left[\left(\phi-\phi_{s}\right) \mathbf{v}\right],
\end{aligned}
$$

where $\frac{\mathrm{d}}{\mathrm{d} t}=\frac{\partial}{\partial t}+\mathbf{v} \cdot \nabla, \mathbf{v}=u \mathbf{i}+v \mathbf{j}$ with $\mathbf{i}, \mathbf{j}, \mathbf{k}$ being the unit vectors in the three orthonormal directions on the sphere, $u$ and $v$ are the zonal and meridional velocity components, respectively, $h$ is the fluid depth, $h_{s}$ is the bottom topography, $g$ the gravitational constant, $\phi=g h, \phi_{s}=g h_{s}$, and $f$ is the Coriolis parameter. The norm used on the state space $\mathbf{x}$ is the total energy norm, induced by the inner product

$$
\langle\mathbf{x}, \mathbf{x}\rangle_{\mathbf{E}}=\frac{1}{2}\left(u^{2}+v^{2}\right)+\frac{g}{h_{0}} h^{2} .
$$

A Godunov type finite volume discretization method is used to discretize the SW equations. The Van-Leer transport scheme, as described in [11, is employed for the space discretization. Computations are done on a $2.5^{\circ} \times 2.5^{\circ}$ grid with a time step $\Delta t=450 \mathrm{~s}$ and the verification time is set at $t_{v}=t_{0}+24 \mathrm{~h}$. The reference state ('truth') $\mathbf{x}_{0}^{t}$ is taken from the trajectory produced by a numerical integration of the SW model using as initial condition the 500hPa ERA-40 data set from the European Centre for Medium-Range Weather Forecasts (ECMWF), valid for March 152002 at 06 : 00 hours. The background state $\mathbf{x}^{b}$ is taken from a 6 -hour model simulation initialized at $t_{0}-6 \mathrm{~h}$ with the ERA-40 data set valid for March 152002 at 00 : 00 hours. The difference between the $24 \mathrm{~h}$ forecasts initiated from $\mathbf{x}_{0}^{t}$ and $\mathbf{x}^{b}$, respectively, exhibits a high discrepancy in the region $\left[55^{\circ} \mathrm{W}, 35^{\circ} \mathrm{W}\right] \times\left[52^{\circ} \mathrm{N}, 65^{\circ} \mathrm{N}\right]$ which is taken as the verification domain $\mathcal{D}_{v}$ at $t_{v}$.

The discrete TLM, FOA, and SOA models are obtained using the Automatic Differentiation package TAMC [12. The discrete SOA can be seen as the action of the Hessian matrix of the scalar forecast aspect of interest on a vector. The code for the second order adjoint can be computed in the forward over reverse mode, this is, taking the tangent of a forward-backward integration.

\subsection{Taylor Approximation with Adjoint Models}

The Taylor approximation (6) is valid for relatively small perturbations of the initial condition $\mathbf{x}_{0}$ and a short forecast time lead, depending on the nonlinearity of the forward model. Taking the SW model as the forward model, forecast perturbations are computed together with their first and second order Taylor 

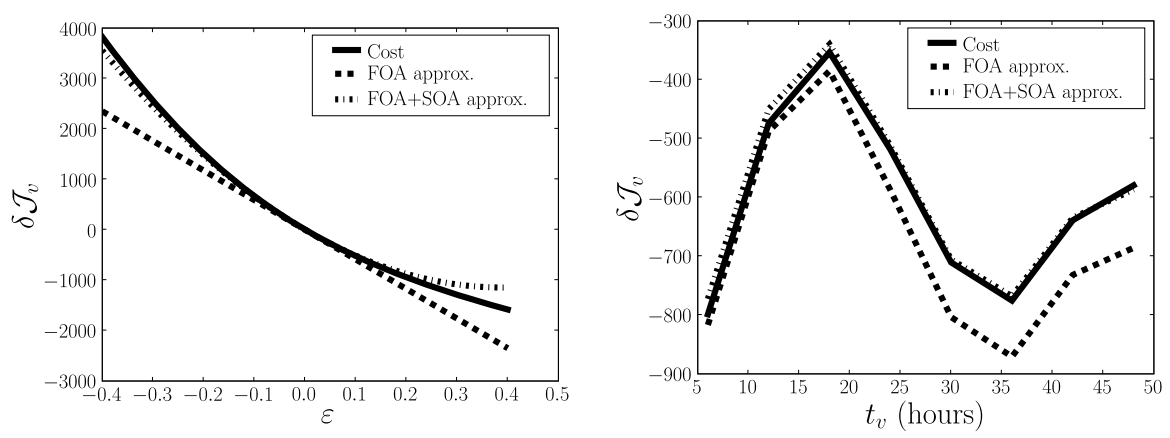

Fig. 1. Left figure: Taylor approximation (6) of the perturbation of the forecast error functional (3) as a function of initial condition perturbations coefficient $\varepsilon$. Right figure: Time evolution of (6) as a function of the verification time $t_{v}$, with $\varepsilon=0.1$.

approximations using the FOA and SOA models. To corroborate the accuracy of the approximations to $\delta \mathcal{J}_{v}$, the initial condition, taken from the background state, is perturbed according to

$$
\mathbf{x}_{0}(\varepsilon)=\mathbf{x}_{0}+\delta \mathbf{x}_{0}(\varepsilon), \quad \delta \mathbf{x}_{0}(\varepsilon)=\varepsilon\left(\mathbf{x}_{0}-\mathbf{x}^{t}\left(t_{0}\right)\right)
$$

where $\varepsilon$ is a coefficient that controls the perturbation in the initial condition of the forward model. The perturbation $\delta \mathcal{J}_{v}$, as well as the adjoint-based approximations, are computed for values of the perturbation coefficient $\varepsilon$ ranging from -0.4 to 0.4 with increments of 0.01 and fixed $t_{v}=24 \mathrm{~h}$, then for a time-varying forecast lead $t_{v}-t_{0}$ ranging from 6 -hour to 72 -hour with one hour increments.

Figure 1 (left) shows the perturbation $\delta \mathcal{J}_{v}$ of the forecast error, and its first and second order Taylor approximations. It is noticed that the second order approximation remains accurate over a wide range of perturbations as compared to the first order approximation.

Figure 1 (right) shows the time evolution of the Taylor approximation using the FOA and SOA models, as a function of $t_{v}$, while keeping $\varepsilon=0.1$ fixed. As the verification time increases the second order approximation remains significantly more accurate than the first order approximation. Similar results were obtained with various values of $\varepsilon \neq 0$.

It must be noticed that the perturbation growth is time dependent, this is, the solutions of the forward and adjoint models depend on the verification time $t_{v}$. An important question to address is the accuracy of the approximation as the verification time is increased. The approximation can lose accuracy if there is a strong nonlinear time dependence of the forward model which is not accurately captured in the adjoint models. Traditional targeting strategies account only for linear error propagation and differ on the selection of the norm used to measure the error growth propagation (e.g. total energy vs. error covariance metric [3]). The limited accuracy of the first order approximation is a major difficulty in 
extending the targeting time interval and the use of second order derivative information may prove to be of relevance in practical applications.

\section{Targeting Using SOA Information}

Applications of the FOA sensitivity analysis during field experiments to collect targeted observations are presented in [1], which gives the fundamentals for targeting strategies based on adjoint modeling.

The FOA model provides the gradient of the forecast error functional $\mathcal{J}_{v}$ with respect to the initial condition $\mathbf{x}_{0}$ of the forward model. The gradient is used to define a space-distributed sensitivity field

$$
F_{v}=\left\|\nabla_{\mathbf{x}_{0}} \mathcal{J}_{v}\right\|_{2}
$$

where the 2-norm is taken at each grid-point on the mesh. In the FOA targeting approach supplementary observations are taken at locations where $F_{v}$ exhibits the largest magnitude.

To accurately track the propagation of perturbations, we propose a new targeting method to incorporate SOA information.

Consider the second term in the Taylor approximation (6)

$$
\frac{1}{2} \delta \mathbf{x}_{0}^{T} \mathbf{H} \delta \mathbf{x}_{0}
$$

where $\mathbf{H}=\nabla_{\mathbf{x}_{0}}^{2} \mathcal{J}_{v}\left(\mathbf{x}_{N}\right)$ is the Hessian matrix of $\mathcal{J}_{v}$. Without loss of generality, consider initial perturbations with unit two-norm, that is $\left\|\delta \mathbf{x}_{0}\right\|_{2}=1$, then (16) is a Rayleigh-Ritz ratio

$$
\frac{\delta \mathbf{x}_{0}^{T} \mathbf{H} \delta \mathbf{x}_{0}}{\delta \mathbf{x}_{0}^{T} \delta \mathbf{x}_{0}} .
$$

The vector for which the Rayleigh-Ritz ratio (17) is maximized provides the direction of maximal quadratic error propagation in the second order term of the Taylor approximation. Since $\mathbf{H}$ is a symmetric matrix, the maximum of (17) is provided by the eigenvector associated with the leading eigenvalue of $\mathbf{H}[13]$.

In [4, 3] the singular vectors of the tangent linear model are used to define a sensitivity field for targeted observations. Following a similar approach, we define the sensitivity function based on the leading eigenvectors of the Hessian.

Let $\sigma_{i}$ be the $i$ th eigenvalue of $\mathbf{H}$, ordered so that $\sigma_{1} \geq \sigma_{2} \geq \ldots \geq \sigma_{n}$, and let $\mathbf{v}_{i}$ be its corresponding eigenvector. Consider the first $m$ leading eigenvectors $\mathbf{v}_{i}, i=1, \ldots, m$, where $m<<n, n$ being the dimension of the matrix. The SOA-based sensitivity field is defined as

$$
F_{m}=\sum_{i=1}^{m} \frac{\sigma_{i}}{\sigma_{1}}\left\|\mathbf{v}_{i}\right\|_{2}^{2},
$$

were the norm of the eigenvector is evaluated on each grid-point of the mesh. 


\subsection{Eigenvectors of the Hessian of the SW Forecast}

At the 2.5-degree grid resolution the dimension of the discrete state vector $\mathbf{x}$ is $n \sim 3 \times 10^{4}$, such that the number of entries in the Hessian matrix $\mathbf{H}$ is of order of $10^{9}$. Storing such a matrix is clearly unpractical, however, iterative methods that require the action of the matrix on a vector can be implemented to obtain the Hessian eigenvalues and eigenvectors. The Arnoldi Package (ARPACK) [14] is used to compute the leading eigenpairs of $\mathbf{H}$. For one matrix vector product of the Hessian the tangent linear model is integrated forward in time to the verification time $t_{v}$ and the FOA and SOA models are integrated backward in time to any targeting instance $t_{i}$ of the data assimilation window.
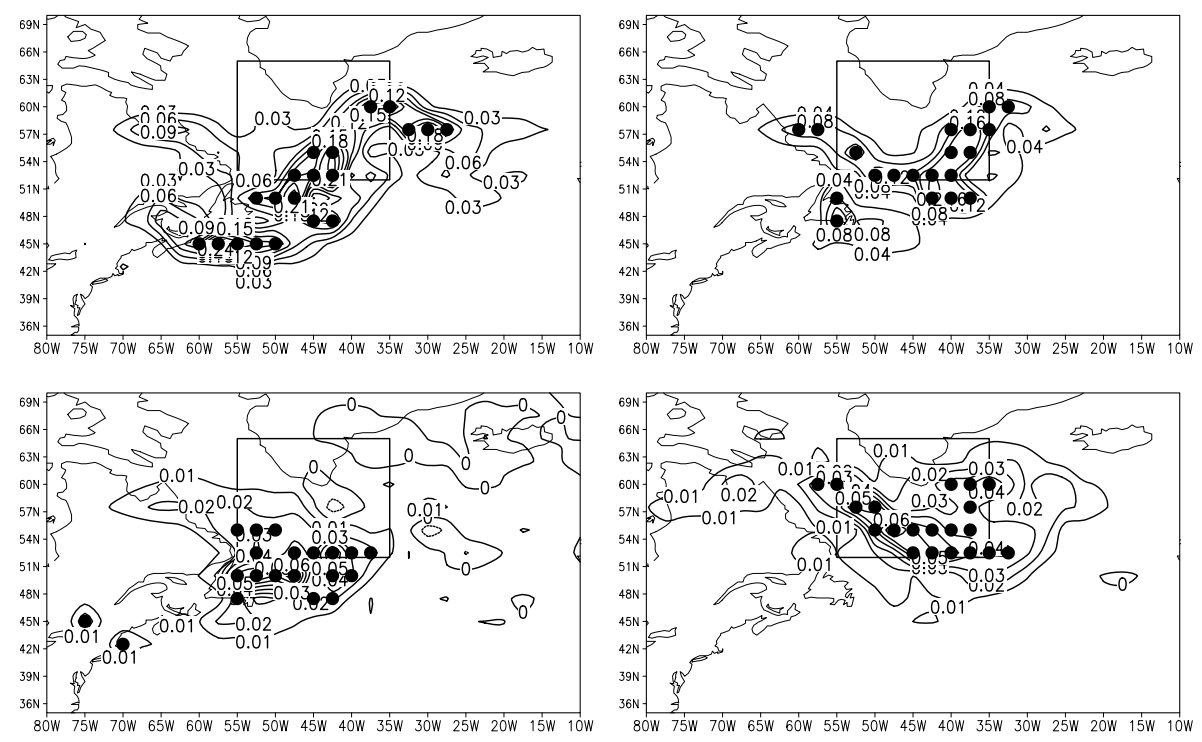

Fig. 2. Top figures: sensitivity field (15) at $t_{i}=t_{0}$ (left) and at $t_{i}=t_{0}+6 \mathrm{~h}$ (right). Bottom figures: sensitivity field (18) with $m=10$ eigenvectors at $t_{i}=t_{0}$ (left) and at $t_{i}=t_{0}+6 \mathrm{~h}$ (right). The verification domain $\mathcal{D}_{v}$ is the region within the rectangle.

The setup for the reference and control simulations, as well as the verification domain and verification time, are the same as in section 3 . For comparison, the sensitivity fields obtained with the FOA sensitivity function (15) are shown in figure 2 for $t_{i}=t_{0}$ (top-left) and $t_{i}=t_{0}+6 \mathrm{~h}$ (top-right). The locations of adaptive observations (marked with $\bullet$ ) correspond to the grid points where the sensitivity fields have the largest magnitude. Figure 2 also shows the sensitivity field obtained with (18), using $m=10$ leading eigenvectors of the Hessian matrix, at $t_{i}=t_{0}$ (bottom-left) and $t_{i}=t_{0}+6 \mathrm{~h}$ (bottom-right). The difference in the sensitivity fields between the FOA and SOA methods illustrates the different type of perturbation growth being measured. Both FOA and SOA fields are time-varying, thus the location of targeted observations depends on the targeting instant. 
Table 1. Leading eigenvalues of the Hessian matrix for $t_{i}=0 \mathrm{~h}$ to $6 \mathrm{~h}$, at 1 -hour increments and corresponding CPU time for the computation of the leading 10 eigenvalues and eigenvectors at each time instance

\begin{tabular}{c|ccccccc}
$t_{i}(\mathrm{~h})$ & 0 & 1 & 2 & 3 & 4 & 5 & 6 \\
\hline$\sigma_{1}$ & 8.455 & 7.272 & 6.721 & 5.932 & 5.270 & 4.355 & 3.541 \\
$\mathrm{CPU}(\mathrm{s})$ & 1698.52 & 1651.28 & 1589.41 & 1542.74 & 1477.11 & 1429.97 & 1368.50
\end{tabular}

Table 1 shows the leading eigenvalue of $\mathbf{H}$ for a targeting time $t_{i}$ from $0 \mathrm{~h}$ to $6 \mathrm{~h}$, at 1 -hour increments. The leading eigenvalue decreases in magnitude as $t_{i}$ increases, which may indicate that as the forecast time lead is shortened the impact of the second order derivative information decreases. This is consistent with the analysis in section 3.1, where the contribution of the SOA was shown to diminish for forecasts closer to the initial time. Table 1 also shows the CPU time, in seconds, for the computation of 10 leading eigenpairs of the Hessian matrix at each hour on a $1.86 \mathrm{GHz}$ Xeon Quad Core 5320 Processor. The computational overhead of a SOA integration is about 3 times that of a FOA integration.

\subsection{Targeted Observations and Data Assimilation Experiments}

To illustrate the SOA targeting strategy we apply a 4D-Var data assimilation scheme with adaptive observations to the SW model. The assimilation window is $[0 \mathrm{~h}, 6 \mathrm{~h}]$ with the verification time set $t_{v}=24 \mathrm{~h}$. A first assimilation experiment is performed with 20 adaptive observations placed at $t=0 \mathrm{~h}$ where the sensitivity field (18) has the highest values, as marked in figure2 (bottom-left). The performance of adaptive observations obtained from the eigenvector sensitivity function (18) is compared with that obtained from the FOA sensitivity function (15), as marked in figure 2 (top-left).
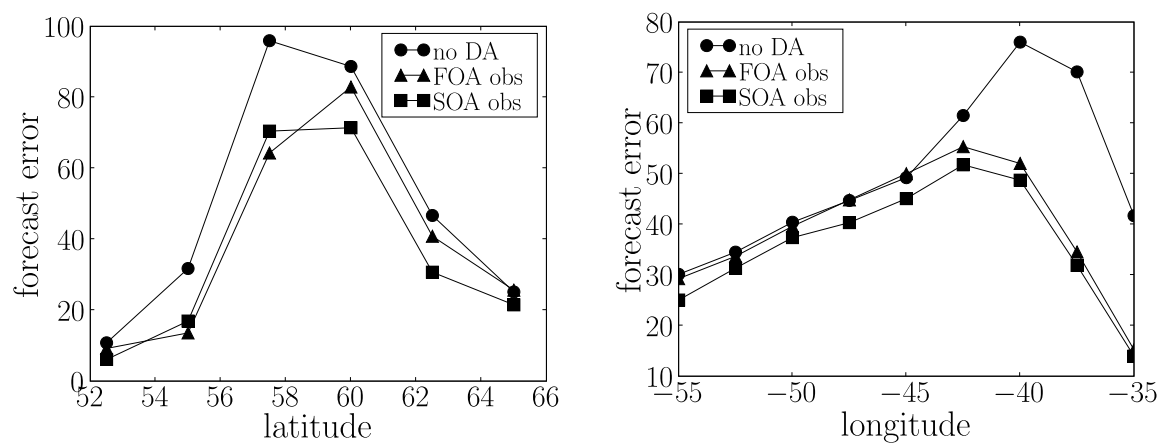

Fig. 3. Longitudinal (left) and latitudinal (right) forecast error average over the verification domain without data assimilation (circles), with data assimilation using adaptive observations from FOA (triangles), and adaptive observations from eigenvectors of the Hessian (squares) 

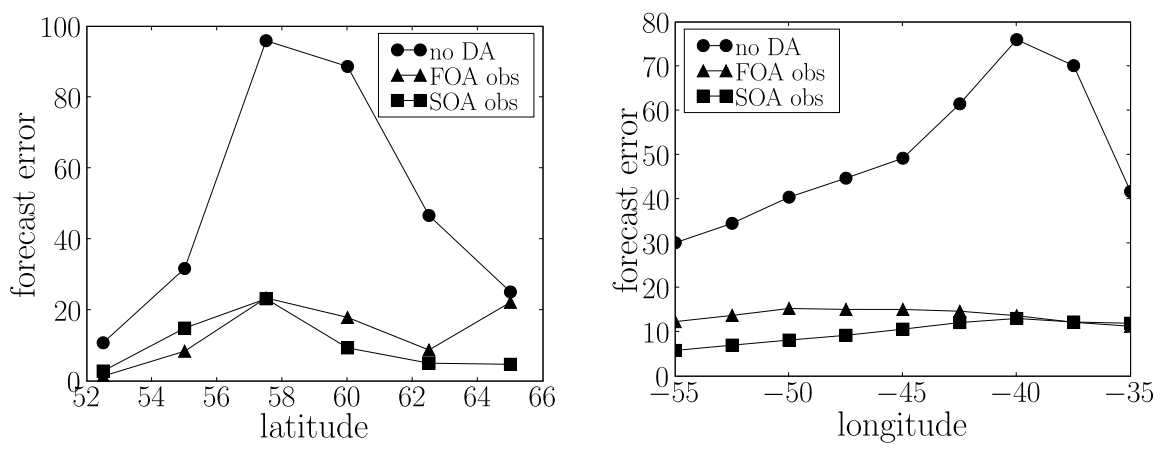

Fig. 4. Longitudinal (left) and latitudinal (right) forecast error average over the target domain without data assimilation (circles), with data assimilation using adaptive observations at both $t=0 \mathrm{~h}$ and $t=6 \mathrm{~h}$ from FOA (triangles), and eigenvectors of the Hessian (squares)

Figure 3 shows the longitudinal (left) and latitudinal (right) forecast error average over the verification domain. It is noticed that the SOA targeting guidance improved the overall forecast quality, as compared to the FOA methodology.

In a second experiment, 20 adaptive observations are placed at both $t=0 \mathrm{~h}$ and $6 \mathrm{~h}$. Figure 4 shows the longitudinal and latitudinal averages of the forecast error, where again it is noticed an improvement in the forecast quality from the adaptive observations obtained with the SOA field (eigenvectors) over the adaptive observations obtained with the FOA sensitivity field. It is also noticed that insertion of targeted observations at $t=6 \mathrm{~h}$ is of significant benefit to the forecast, indicating a larger forecast impact from these observations. This is consistent to the observation sensitivity study in [6] where is was found that the forecast sensitivity to observations increases for observations near the end of the assimilation window, and thus closer to the verification time. In addition, accounting for data interaction is essential when multiple targeting instants are considered [15, and this is an area where further research is much needed. Nevertheless, the results show the relevance of the SOA in targeting strategies when dealing with large time intervals and/or highly nonlinear forecast models.

\section{Conclusions}

Properly accounting for nonlinear error growth is an unresolved issue in targeted observations for numerical weather prediction. In this study a novel approach based on second order adjoint modeling is proposed to account for the quadratic initial-condition error growth in the model forecast. Preliminary numerical experiments indicate that the SOA methodology is effective and may outperform the traditional FOA approach to observation targeting. Further experiments are required to validate this approach in realistic models. To fully exploit the benefits of the SOA model, novel targeting strategies must combine information from both FOA and SOA models to form a more cohesive and accurate strategy. 
Acknowledgments. This research was supported by the NASA Modeling, Analysis and Prediction Program under award NNG06GC67G and by the 2006 Intel Oregon Faculty Fellowship program. The work of the first author was also supported by a scholarship from Consejo Nacional de Ciencia y Technologìa.

\section{References}

1. Langland, R.H., Gelaro, R., Rohaly, G.D., Shapiro, M.A.: Targeted observations in FASTEX: Adjoint-based targeting procedures and data impact experiments in IOP17 and IOP18. Q. J. R. Meteorol. Soc. 125, 3241-3270 (1999)

2. Langland, R.H.: Issues in targeted observing. Q. J. R. Meteorol. Soc. 131, 34093425 (2005)

3. Palmer, T.N., Gelaro, R., Barkmeijer, J., Buizza, R.: Singular Vectors, Metrics, and Adaptive Observations. J. Atmos. Sci. 55, 633-653 (1998)

4. Buizza, R., Montani, A.: Targeting Observations Using Singular Vectors. J. Atmos. Sci. 56, 2965-2985 (1999)

5. Baker, N.L., Daley, R.: Observation and background adjoint sensitivity in the adaptive observation-targeting problem. Q. J. R. Meteorol. Soc. 126, 1431-1454 (2000)

6. Daescu, D.N.: On the Sensitivity Equations of Four-Dimensional Variational (4DVar) Data Assimilation. Mon. Wea. Rev. 136, 3050-3065 (2008)

7. Le Dimet, F.X., Navon, I.M., Daescu, D.N.: Second-Order Information in Data Assimilation. Mon. Wea. Rev. 130, 629-648 (2002)

8. Kalnay, E.: Atmospheric Modeling, Data Assimilation, and Predictability, p. 364. Cambridge University Press, Cambridge (2002)

9. Le Dimet, F.X., Talagrand, O.: Variational algorithms for analysis and assimilation of meteorological observations: theoretical aspects. Tellus 38A, 97-110 (1986)

10. Daescu, D.N., Navon, I.M.: Efficiency of a POD-based reduced second-order adjoint model in 4D-Var data assimilation. Int. J. Numer. Meth. Fluids 53, 985-1004 (2007)

11. Lin, S.J., Chao, W.C., Sud, Y.C., Walker, G.K.: A Class of the van Leer-type Transport Schemes and Its Application to the Moisture Transport in a General Circulation Model. Mon. Wea. Rev. 122, 1575-1593 (1994)

12. Giering, R.: Tangent linear and Adjoint Model Compiler, Users manual. Center for Global Change Sciences, Department of Earth, Atmospheric, and Planetary Science. MIT, Cambridge (1997)

13. Horn, R.A., Johnson, C.R.: Matrix Analysis. Cambridge University Press, Cambridge (1985)

14. Lehoucq, R.B., Sorensen, D.C., Yang, C.: ARPACK Users' Guide: Solution of Large-scale Eigenvalue Problems with Implicitly Restarted Arnoldi Methods. Society for Industrial and Applied Mathematics, Philadelphia (1998)

15. Daescu, D.N., Navon, I.M.: Adaptive observations in the context of 4D-Var data assimilation. Meteorol. Atmos. Phys. 85, 205-226 (2004) 